International Journal of Advanced Technology in Mechanical, Mechatronics and Materials

(IJATEC)

Vol. 01, No. 3 (2020) 83-89

Institute for Research on Innovation and Industrial System (IRIS)

\title{
Design and Build A Plastic Pellet Monitor System Prototype on An Injection Molding Plastic Storage Tank with The Blynk Application
}

\author{
Agus Dendi Rochendi ${ }^{*}$, Irvan Kampono ${ }^{b}$ \\ ${ }^{a}$ Department of Electrical Engineering, Faculty of Engineering, Universitas Mercu Buana, Jakarta, Indonesia \\ ${ }^{b}$ Department of Instrumentation, Indonesian Institute of Sciences, Indonesia
}

\begin{abstract}
In plastic injection molding machines, the plastic pellet filling process is generally done manually in a closed tank. The operator is facing some difficulties in measuring the level of plastic pellets in the storage tank, and as a result, it can slow down productivity. In this research, the prototype of the monitoring system was employed to measure the volume of plastic pellets in the storage tank using the HC-SR04 ultrasonic sensor ESP8266 data processing equipped with LCD and data communication media. After some measurement, the results show that the plastic pellet tank monitor system can satisfy the expectation. After data validation was carried out, it is found that the average accuracy of the ultrasonic sensor 1 is $97.2 \%$, and the average accuracy of the ultrasonic sensor 2 is $95.3 \%$. In the experiment, the ultrasonic sensor readings show the minimum level is $0 \mathrm{~cm}$ and the maximum level is $32.8 \mathrm{~cm}$. Furthermore, it takes an average of 1.9 seconds from reaching the minimum limit until a notification appears on the Blynk application.
\end{abstract}

Keywords: ultrasonic sensor; plastic injection molding; Blynk application

DOI: $10.37869 /$ ijatec.v1i3.29

Received 28 August 2020; Accepted 14 December 2020; Available online 31 December 2020

(c) The Authors. Published by IRIS. This is an open access article under the CC BY-NC-SA license

\section{Introduction}

Injection molding is a process that similar to a syringe, where melted plastic is injected into a tightly closed mold inside the machine so that the material fills the space in the mold according to the desired product shape. The cycle process for injection molding consists of four stages: clamping before injecting the material into the mold. Two parts of the mold must be tightly closed on the machine, and liquid plastic injection is injected into the mold. It fills the room according to the desired product shape, and cooling is a cooling process. After the injection process, plastic material ejection when the mold has opened the mechanism used for the ejection system is to push the cold part of the plastic from the mold. This process is often fully automated [1]. The plastic injection molding machine control system consists of a system drive and an electronic drive. There are three forms of electronic controllers, which include traditional relay controllers, PLC controllers, and microcomputer controllers. Standard relay controllers are not very popular nowadays for monitoring system application due to hardware complexity, low precision, and difficulty adjusting [2].

The plastic injection process takes place on the closed molding. Thermoplastic granules were fed through a holding tank (hopper) into a screw-like plastic barrel where the plastic melted (figure 1). The melted plastic was then injected into a heated mold where the portion is formed. The process is mostly completed automatically [3]. The plastic pellet storage tank was not equipped with a volume indicator for measurement. Generally, filling the material or plastic pellet is done manually on each machine. It is a potential problem because the machine operator does not know whether the tank is full or empty. The delay in filling causes the production process can be interrupted. 


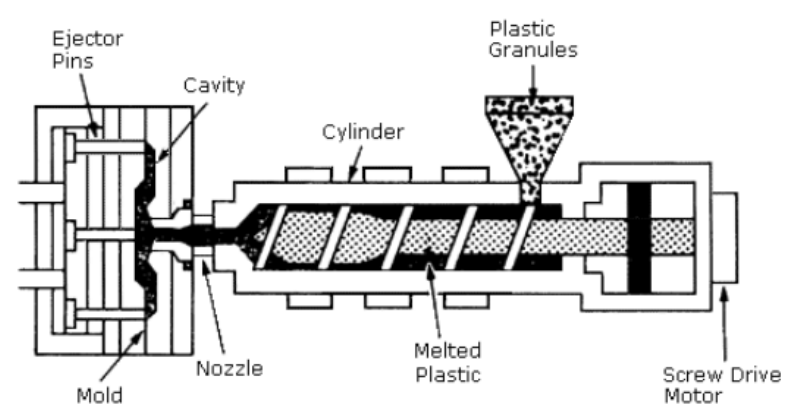

Figure 1. Schematic diagram of a typical injection molding process [3]

The research carried out is designing a prototype tool for monitoring the movement of plastic pellet on the tank using Node MCU ESP8266, which is an IoT (Internet of Things) module [4], a technological connection in which physical objects are used to communicate and react that are connected continuously, especially machine-to-machine communications [5]. IoT platform has proved that by implementation in various fields, it can help vastly resolve problems faced in industries easily [6]. IoT is essential to meet accessibility in both physical and environments every day [7]. The respective data are extracted, stored, and uploaded to the cloud by the implementation of IoT [8].

The ultrasonic sensor HC-SR04 works on the principle of sound waves and applies the law of wave reflection. The angle of incidence of the wave is the same as the angle of reflection of the wave, so that distance can be determined by manipulating the time data into a distance [9] and the Blynk app. so that it can inform the operator quickly about the tank conditions periodically. The working of Blynk app is represented in figure 2. In the application, a button widget is placed on the screen and is configured as a virtual pin [10]. Node MCU ESP8266 acquired the data obtained from the HC-SR04 ultrasonic sensor, which is how to measure contactless distance. The choice of sensor distance measurement to apply it in any application is very important to avoid invalid experimental results. Measurement using an ultrasonic sensor is the cheapest among various options [11]. Then the data that is processed into information can be monitored using the Blynk application [12].

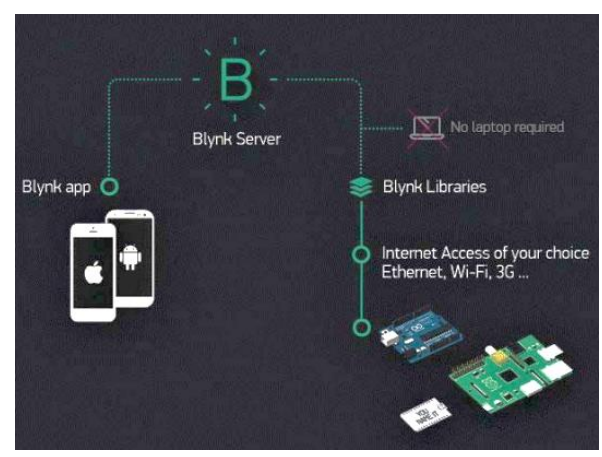

Figure 2. working of Blynk app

\section{Research Methodology}

The shape of the plastic pellet tank is generally in the form of a funnel, which is as shown in Figure 3 and 4.

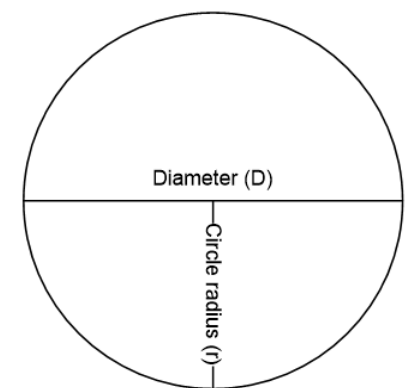

Figure 3. Top of funnel as Circle 


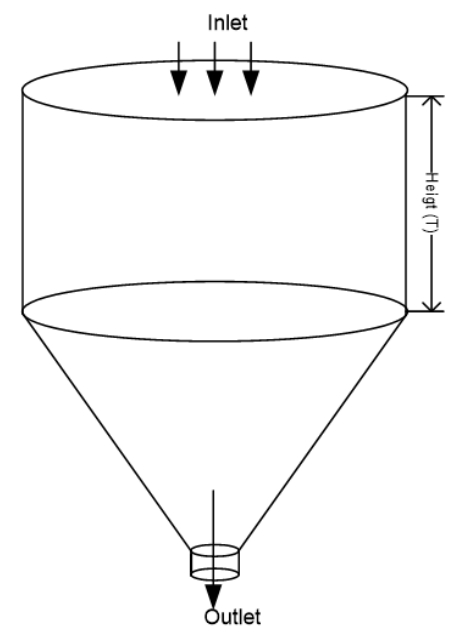

Figure 4. Illustration as a funnel with the top is a cylinder

Volume $=\left(\pi \cdot r^{2}\right) \cdot T$

Where $\pi=22 / 7=3.14 ; \mathrm{r}=$ circle radius; $\mathrm{T}=$ height. The upper surface of the tank was assumed to be the upper limit of filling plastic pellets, and the lower limit of the surface of the cylinder (before) is the upper cone, as shown in Figure 5.

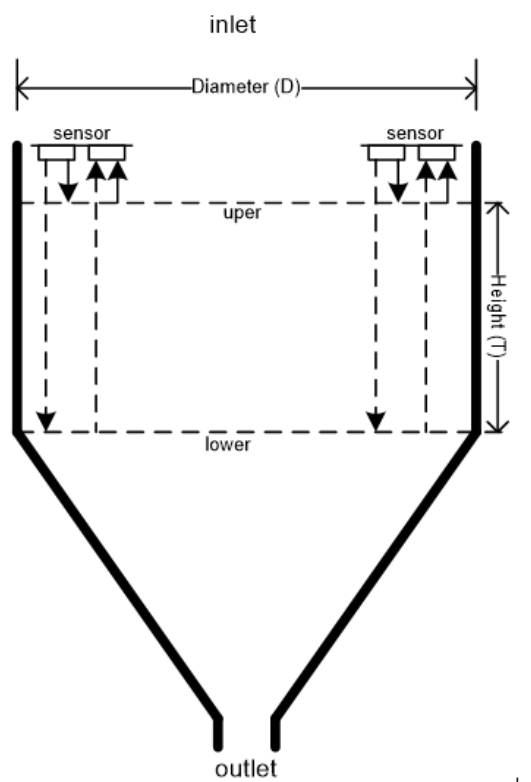

Figure 5. Ultrasonic sensor HC-SR04

The HC-SR04 ultrasonic sensor was installed at the top of the tank to measure the plastic pellet's height, and the plastic pellet height is a variable $\mathrm{T}$, which can be manipulated into a volume value. The flow diagram of a prototype of the tank monitoring tool is as shown in Figure 5.

When the system is activated, the ESP 8266 MCU node will be conditioned to read the initial data obtained from the HC-SR04 ultrasonic sensor displayed on the LCD and the data will be sent with an internet connection to the Blynk application. Blynk is a hardware-agnostic IoT platform with whitelabel mobile applications, private cloud, device management, data analytics, and machine learning [14]. Internet of Things (IoT) is a network of interconnected devices embedded with electronics, software, sensors, and an internet connection to enable these devices to collect and exchange data [15]. If the reading of the ultrasonic sensor data above is equal to $20 \mathrm{~cm}$, it is instructed to activate the LED and Buzzer, and in the Blynk application, a warning message is generated. The installation of two ultrasonic sensors is for safety reasons in case of reading errors. 


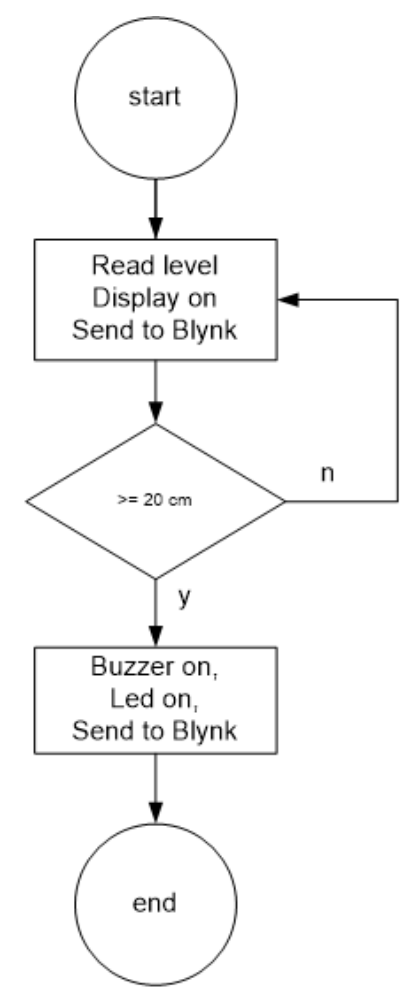

Figure 6. Flowchart of measurement process

\section{Results and Discussions}

The results of the research are presented in graphical or descriptive form. Analysis and interpretation of these results is required prior to discussion.

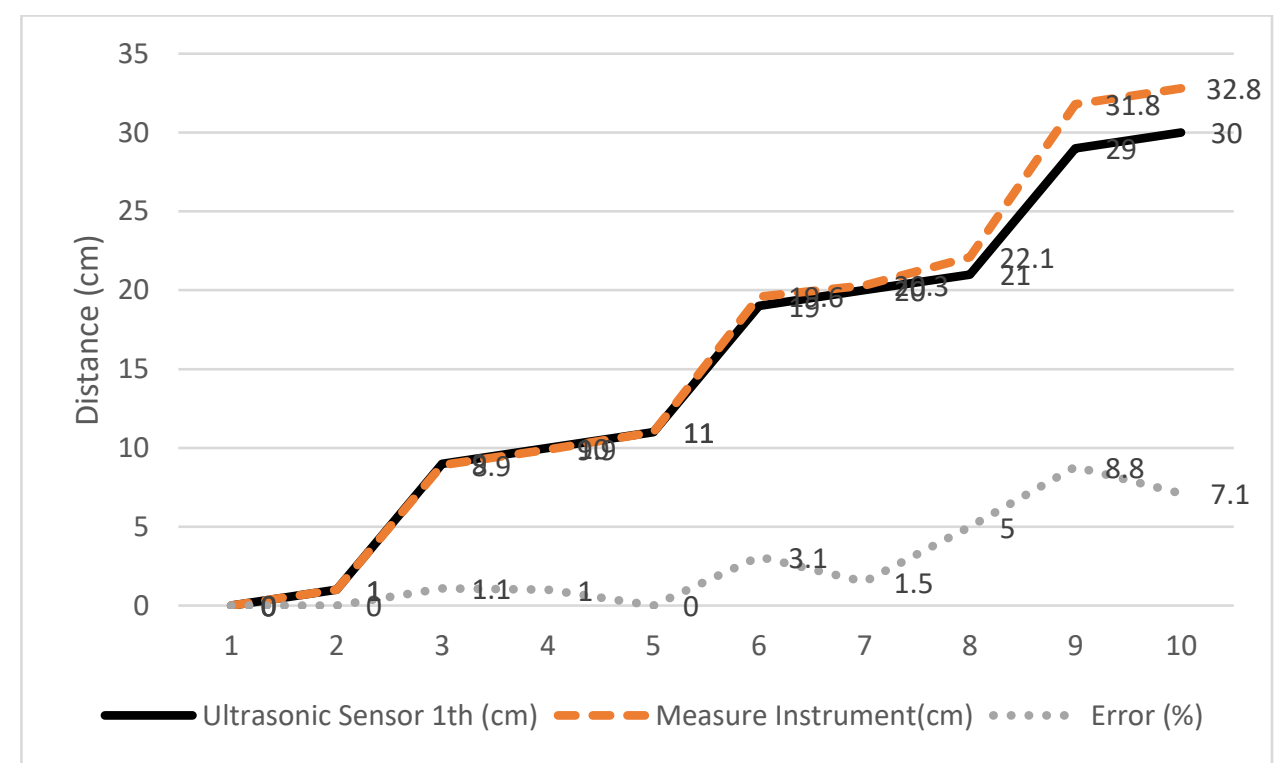

Figure 7. Graph 1 of sensor 1 measurement

It can be concluded that the value of the ultrasonic sensor error range 1 is 0 to $8.8 \%$. The average ultrasonic sensor error 1 was $2.8 \%$, and the ultrasonic sensor 1 accuracy average was $97.2 \%$. The value of the ultrasonic sensor error range 2 is 0 to $11.1 \%$. The average ultrasonic sensor error 2 is $4.7 \%$, and the average accuracy of the ultrasonic sensor 2 is $95.3 \%$.

In Figure 7, the Ultrasonic Sensor Testing Graph 1 shows the information on the measurement distance of the ultrasonic sensor1, the measurement distance with a ruler, and the percentage of 
ultrasonic sensor measurement error 1 . The lowest error percentage is in the 1st, 2nd, and 5th experiments. The highest percentage of errors is in the 9th experiment. Furthermore, the higher the measurement distance of the ultrasonic sensor, the greater the error percentage.

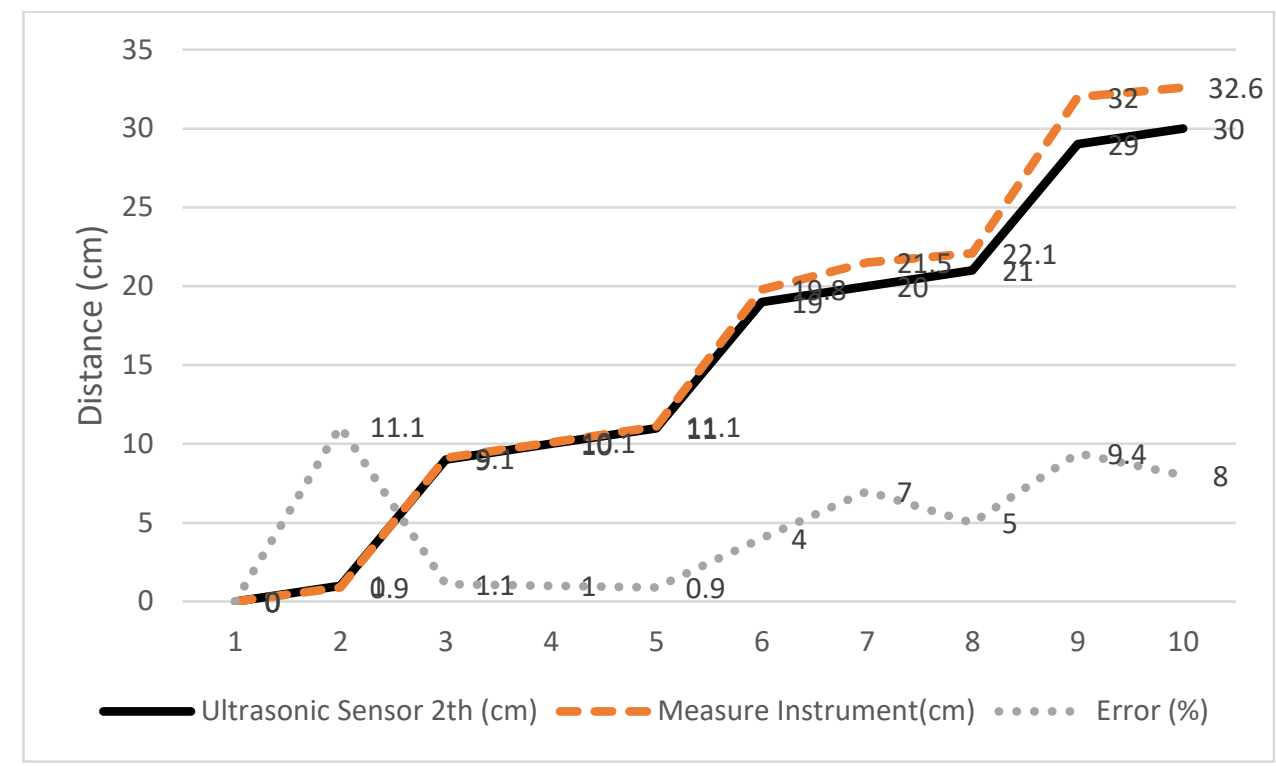

Figure 8. Graph 2 of sensor 2 measurement

In Figure 8, the Ultrasonic Sensor Test Graph 2 can be seen information on the measurement distance of the ultrasonic sensor 2, the measurement distance with a ruler, and the percentage of ultrasonic sensor measurement error 2 . The lowest error percentage is in the 1st experiment. The highest percentage of errors is in the second experiment. In addition, the higher the measurement distance of the ultrasonic sensor 2 , the relatively greater the error percentage.

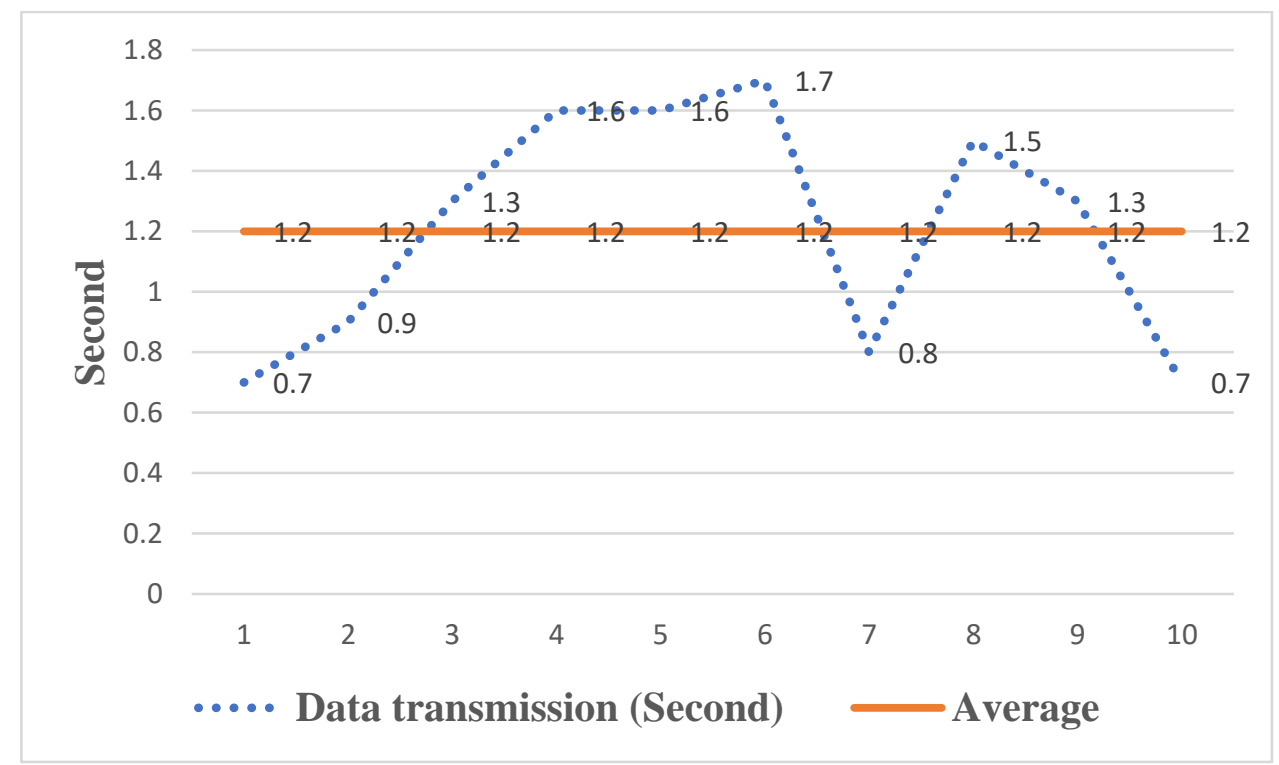

Figure 9. Graph of data send time

It can be concluded that the value range for data transmission speed is 0.7 to 1.7 seconds. The average data transmission speed is 1.2 seconds. Data values are not affected by fast or slow data transmission. In Figure 9, the Data Sending Speed Testing Graph shows the information about data transmission speed and average speed of the measurements. The average speed obtained in 1.2 seconds, and the fastest data transmission time is on the 1st and 10th experiments. The time for sending data was late, namely in the 6th trial. 


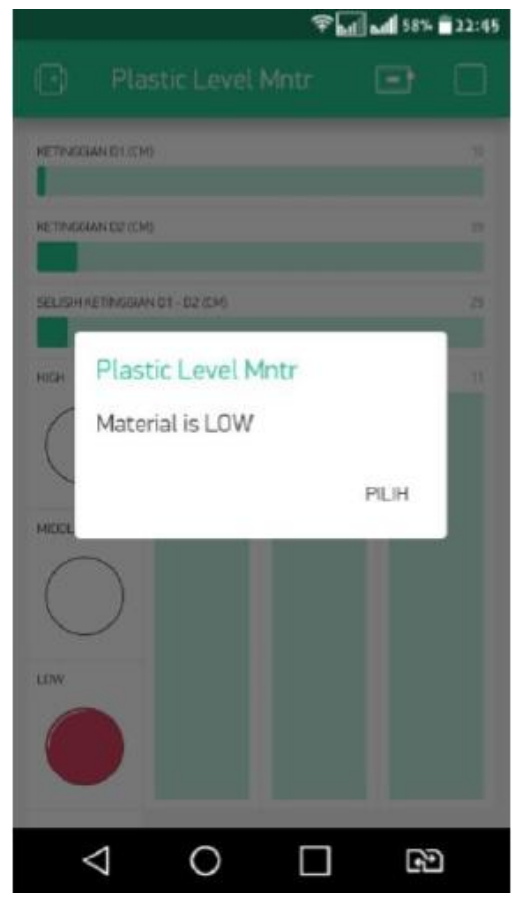

Figure 10. Warning on the Blynk application

Figure 10 shows the Blynk Application Notification Display on the smartphone screen. The notification is still displayed even though the application is not running. When an incoming signal informs that the plastic pellet level is low, the red button indicator is blinking on the Blynk application display on the smartphone screen.

\section{Conclusions}

A prototype of a plastic pellet level monitoring system has been developed to be used on the storage tank for the plastic injection molding machine by utilizing Blynk application. The air quality measurement device was installed on the ESP8266 board. MCU node is connected to 2 ultrasonic sensors, ESP-8266, to detect the plastic pellet level in the storage tank. The average accuracy of the ultrasonic sensor 1 is $97.2 \%$, and the average accuracy of the ultrasonic sensor 2 is $95.3 \%$. The ultrasonic sensor measurements show the minimum level is at $0 \mathrm{~cm}$, and the maximum level is 32.8 $\mathrm{cm}$. It takes an average of 1.9 seconds to reach the top level, starting from the lower limit until a notification appears on the Blynk application. For future developments, the prototype can be integrated with a more complex interface and functions.

\section{References}

[1] Bryce D. M., Plastic Injection Molding Mold Design and Construction Fundamentals, Society of Manufacturing Engineers, Dearborn, Michigan, 1998.

[2] Tan Siyun, Design on Controller of Plastic Injection-molding Machine Based on Multi-CPU, 2010 2nd Conference on Environmental Science and Information Application Technology (ESIAT 2010), (C)2010 IEEE.

[3] OSHA (Occupational Safety and Health Administration) Technical Manual, Polymer Matrix Materials: Advanced Composites, USA Department of Labour 2020, available: https://www.osha.gov/dts/osta/otm/otm_iii/otm_iii_1.html\#8

[4] Lakshmi Goswami , IOT based Diagnosing of Fault Detection in Power Line Transmission through GOOGLE Firebase database, IEEE Proceedings of the Fourth International Conference on Trends in Electronics and Informatics (ICOEI 2020)

[5] Er. Pooja Yadav, IoT: Challenges and Issues in Indian Perspective, IEEE 2018.

[6] Mayuresh Kharade, A Node MCU based Fire Safety and Air Quality Monitoring Device, 2020 International Conference for Emerging Technology (INCET) Belgaum, India. Jun 5-7, 2020. 
[7] Ahmad Kamal Pasha Mohd Daud, An IoT-Based Smart Aquarium Monitoring System, downloaded on June 29,2020 at 06:46:56 UTC from IEEE Xplore.

[8] K. Sai Nikhilesh, Low-cost IoT based weather monitoring system for smart community, Proceedings of the Fourth International Conference on Inventive Systems and Control (ICISC 2020).

[9] Haliday resnick, pantur silaban, Fisika dasar jilid I, erlangga 1980.

[10] J. P. Guruprasadh et al., "Intelligent soil quality monitoring system for judicious irrigation," International Conference on Advances in Computing, Communications and Informatics (ICACCI), pp. 443-448, Udupi, 2017.

[11] Ahmad Kamal Pasha Mohd Daud, An IoT-Based Smart Aquarium Monitoring System, downloaded on June 29,2020 at 06:46:56 UTC from IEEE Xplore.

[12] Nor Anum Zuraimi Md Noar, The Development of Smart Flood Monitoring System using Ultrasonic sensor with Blynk Applications, Proc. of the 4th IEEE International Conference on Smart Instrumentation, Measurement and Applications (ICSIMA) 28-30 November 2017, Putrajaya, Malaysia.

[13] Clemens, Stanley R. Cs., Geometry., USA: Addison-Westley Publishing Company, inc. (1984).

[14] Blynk. 2020. "We make Internet of Things simple"[Online] https://blynk.io/, [Accessed:19Agustus-2020].

[15] Guruprasadh JP, Harshananda A, Intelligent Soil Quality Monitoring System for Judicious Irrigation, (C)2017 IEEE. 